\title{
EDITORIAL
}

\section{Global implications of rising rates of cesarean sections and late preterm births}

\section{Journal of Perinatology (2007) 27, 737; doi:10.1038/sj.jp.7211824}

The study by Almeida et al., is a well-designed prospective cohort study aimed at identifying modifiable risk factors associated with the need for resuscitative procedures in late preterm infants. Although the participating centers were not randomly selected, the investigators made a special effort to include a study sample as representative, as possible, of the population of late-preterm infants in Brazil. Because cesarean section (CS) and late-preterm infant birth rates have been increasing worldwide, and recent studies from both developed and developing countries suggest an increase in associated morbidity, ${ }^{1,2}$ the present study is timely and of significant importance.

Of the total number of late-preterm infants identified during the study period, $45 \%$ were born by CS. A similar rate was observed in the full-term infant population. This number is far greater than recommended by the World Health Organization $(15 \%)^{3}$ and is not explained alone by the described medical indications for CS.

Elective CS played an important role in the high rates observed and require further evaluation to determine what percent were the result of previous non-medical indications.

Ronsmans et al., ${ }^{4}$ evaluating socioeconomic differentials in cesarean rates in 42 developing countries found a positive correlation between CS rate and socioeconomic status. While CS rates $<5 \%$ were observed among $27 / 42(64.2 \%)$ of the countries studied, on the other side of the spectrum, Colombia, Dominican Republic and Brazil had CS rates $>25 \%$. Brazil's overall CS rate was $36 \%$ with a range between the poorest and the richest quintile of 16.4 and $67.6 \%$. In contrast to other developing countries, Brazil's excessive rates of CS are concentrated in the middle and richest quintiles, while recommended rates were observed in the poorest quintile.

Explanations for the observed excess and specific pattern of CS rates in Brazil may include: high rates of adolescent pregnancies (24\%) and non-medical determinants such as financial incentives, malpractice litigation, convenience of the physician and women's choice. ${ }^{4}$ Whatever the underlying causes for this phenomena, the present study highlights the increased morbidity associated with delivery of late-preterm infants and the potential for long-term consequences. Positive pressure ventilation in the study population was $14 \%$, a rate more than twice as high as that observed in their population of term infants $(6 \%)$. Of the risk factors identified as independently associated with the need for positive pressure ventilation, maternal hypertension, CS and gestational age have the greatest potential to be modified through evidence-based interventions.

Supporting the results of the present study, Villar et al., ${ }^{1}$ found that rate of CS in Latin America was positively associated with postpartum antibiotic treatment and severe maternal morbidity and mortality. After adjustment for preterm delivery, increased CS rates were also associated with increased fetal mortality rates and higher number of babies admitted to intensive care for 7 days or longer. Althabe et al., ${ }^{5}$ found similar results in medium-income countries. The general estimates are that $50 \%$ of infants born at 34 weeks' gestation require intensive care; this number drops to $8 \%$ at 36 weeks. ${ }^{2,6}$

Retrospective and prospective epidemiologic data from around the world highlight an increase trend in rates of CS that do not meet the demands of the poorest and most vulnerable populations and increases the risk of enhanced mortality and morbidity in low-risk populations. The current trend is an example of both local and global misuse of healthcare resources. Research aimed at decreasing the number of late-premature births secondary to non-medical indications remains a formidable challenge. ${ }^{7}$

MA Rojas
Division of Neonatology, Department of Pediatrics, Vanderbilt
University, Nashville, TN, USA
E-mail: mario.a.rojas@vanderbilt.edu

\section{References}

1 Villar J, Valladares E, Wojdyla D, Zavaleta N, Carroli G, Velazco A et al. Caesarean delivery rates and pregnancy outcomes: the 2005 WHO global survey on maternal and perinatal health in Latin America. Lancet 2006; 367: 1819-1829.

2 Jain L. The late preterm infant. Neonatology Today 2007; 2: 1-9.

3 Belizán JM, Althabe F, Cafferata ML. Health consequences of increasing caesarean section rates. Epidemiology 2007; 18: 485-486.

4 Ronsmans C, Holtz S, Stanton C. Socioeconomic differentials in caesarean rates in developing countries: a retrospective analysis. Lancet 2006; 368: 1516-1523.

5 Althabe F, Sosa C, Belizán JM, Gibbons I, Jacquerioz F, Bergel E et al. Cesarean section rates and maternal and neonatal mortality in low, medium, and high income countries: an ecological study. Birth 2006; 33: 270-277.

6 Clark RH. The epidemiology of respiratory failure in neonates born at an estimated gestational age of 34 weeks or more. J Perinatol 2005; 25: 372-376.

7 Althabe F, Belizán JM, Villar J, Alexander S, Bergel E, Ramos S et al. Mandatory second opinion to reduce rates of unnecessary caesarean sections in Latin America: a cluster randomized controlled trial. Lancet 2004; 363: 1934-1940. 